\title{
Importancia del marcado de la prótesis dentaria en el reconocimiento de personas: descripción de dos técnicas
}

\author{
Important the marking of dental prosthesis in the recognition of people: description of two \\ techniques
}

Gabriela Aracely Sanchez- Sandoval ${ }^{1 a}$, Jessica Figueroa-Contreras ${ }^{2 a}$

\section{RESUMEN}

El reconocimiento de personas que pierden la vida en desastres naturales o accidentes se complica cuando las víctimas son edéntulos totales, por ende, la necesidad de buscar nuevas formas de identificación que sean confiables y fáciles de ser implementadas. Teniendo en cuenta esta premisa, se realizó una búsqueda de la información de la literatura con el objetivo de demostrar que el marcado de prótesis dentaria es importante en el reconocimiento de personas y que puede ser implementado por el gobierno del Perú. Para lo cual se eligieron tres técnicas de marcado siendo: Código QR, Tarjetas digitales e Incorporación de banda metálica. Se procedió a realizar una selección rigurosa de la información en las bases de datos Pubmed, Scielo y BVS (Biblioteca Virtual en Salud) de artículos publicados entre los años 2012-2019, en idioma español e inglés. Se encontraron 105 artículos de los cuales se escogieron solo 18 de ellos, que cumplían con los criterios de inclusión. Se concluye que esta técnica es importante en la identificación de personas, y se sugiere considerar su implementación en nuestro país. Al final se presentan dos técnicas de marcado de prótesis en modelos tomados a un simulador.

PALABRAS CLAVE: Dentadura completa; Odontología forense; Identificación de la prótesis; Etiquetas de prótesis .

\section{ABSTRACT}

The recognition of people who lose their lives in natural disasters or accidents are complicated when the victims are total edentulous, therefore, the need to look for new forms of identification that are reliable and easy to be implemented. Taking into account this premise, a search of the information in the literature was carried out in order to demonstrate that the marking of dental prostheses is important in the recognition of people and that they can be implemented by the government of Peru. For which three marking techniques were chosen: QR code, Digital cards and Incorporation of metal band. A rigorous selection procedure of the information was carried out in the databases, Pubmed, Scielo and VHL (Virtual Health Library), with articles published between 2012-2019, in Spanish and English. 105 articles were found of which only 18 of them were chosen, which met the inclusion criteria. It is concluded that this technique is important in the identification of people, and it is suggested to consider its implementation in our country. At the end, two prosthesis marking techniques are presented on models taken from a simulator.

KEY WORDS: Denture complete; Forensic Dentistry; Denture Identification; Marking dentures

\footnotetext{
${ }^{1}$ Universidad de San Martin de Porres, Facultad de

Odontología. Lima, Perú.

a Bachiller

${ }^{\mathrm{b}}$ Docente del área de Rehabilitación Oral

\section{Correspondencia:}

Gabriela Aracely Sánchez Sandoval

Dirección: Coop Las Palmeras M “C” Lt 12

Teléfono: 0969791021

Correo electrónico: gabrielasanchezsand@gmail.com
}

Este es un artículo Open Access distribuido bajo la licencia Creative Commons Atribución-No

Comercial-Compartir Igual 4.0

Citar como: GA Sánchez, J Figueroa. Importancia del marcado de la prótesis dentaria en el reconocimiento de personas: descripción de dos técnicas . KIRU. 2020;17(2): 99-106 https://doi.org/10.24265/kiru.2020.v17n2.07 


\section{INTRODUCCIÓN}

El reconocimiento de personas se complica cuando se enfrenta a diversos tipos de accidentes o desastres donde la pérdida de vida es cuantiosa. En estos casos el reconocimiento de las víctimas se dificulta más aún, cuando estas son desdentadas totales o parciales ${ }^{(1,2)}$.

El 29 de diciembre del 2001 se suscitó un incendio en un sector del centro de Lima, en este siniestro se perdieron muchas vidas, solo 300 personas fueron reconocidas, 350 quedaron como desaparecidas y ademas hubo más de 600 heridos. El reconocimiento de las víctimas se dio por algún objeto encontrado que pudo ser identificado por sus familiares, los demás cadáveres no pudieron ser reconocidos. Las víctimas presentaban restauraciones dentales 0 algún tipo de prótesis dental total o parcial, algunos de estos tratamientos resistieron al fuego, pero no sirvió para el reconocimiento, pues no brindaban información alguna. Sin embargo, si estas prótesis dentales se hubieran encontrado marcadas, hubiera podido reconocerse a más víctimas de este siniestro $(3,4)$.

La identificación es un derecho que tienen todos los individuos de una sociedad, con propósitos legales, sociales y humanitarios (5). Al encontrar la odontología forense esta problemática, busca métodos que permitan el reconocimiento de las personas. Keiser-Nielsen, citados por Rao 2016, definen a la odontología forense como "esa rama de la odontología, en interés de la justicia, relacionada con el manejo, el examen, la identificación y la presentación de hallazgos dentales" (6).

Con esta premisa, se ha valorado el marcado de las prótesis dentales, porque puede ofrecer información para el reconocimiento rápido de los individuos, a través de datos como: número de tarjeta de identidad, número de seguro social, número de licencia de conducir, fecha de nacimiento, sexo y otros ${ }^{(7)}$ y mediante el uso de técnicas más complejas como el uso códigos 2D de barras y microchips, que permiten acceder a la historia odontológica y médica del paciente ${ }^{(8)}$.En la literatura se han encontrado diferentes técnicas de marcado que pueden ser utilizadas por los odontólogos e implementadas por los gobiernos, dependiendo de los beneficios que estas puedan brindar ${ }^{(9)}$. Se refiere que el marcado de prótesis dentales esta regulado en países como Suecia, Islandia y en 21 estados de Estados Unidos de América ${ }^{(2)}$.

Algunas de estas son: Las técnicas de marcado superficial de prótesis dentales con códigos de identificación y las técnicas de marcado por inclusión, que incorporan a la estructura de las prótesis dispositivos con información del paciente como: bandas metálicas con códigos de identificación (ID-Ban); etiquetas de papel impresas con códigos de barras; memorias SD con información digitalizada y microchips electrónicos con códigos de identificación digital ${ }^{(2)}$. La prótesis dental puede ser marcada durante o después de la fabricación.

Las técnicas de marcaje pueden ser diversas, pero en este trabajo solo nos enfocaremos en tres: Método de Identificación mediante Código $Q R$, Tarjetas digitales, incorporación de Banda metálica (10). Asimismo, solo se eligieron estas tres, luego de evaluar sus ventajas y desventajas y la viabilidad de poder ser implementadas por el gobierno peruano e instituciones privadas como clínicas odontológicas universitarias.

A esta revisión se agrega la descripción de dos técnicas Códigos QR y tarjetas digitales, y se explica su elaboración. El proceso se realizó en dos juegos de prótesis completa, los cuales fueron tomados a un simulador dental.

El gobierno podría considerar la obligatoriedad de incorporar sistemas de marcación en las prótesis completas o parciales como medida de prevención. Estas podrían ser implementadas como proyectos pilotos en las clínicas odontológicas de las universidades.

El objetivo de este trabajo es mostrar que el marcado de prótesis dental es importante en el reconocimiento de personas y que podría ser implementada por entidades gubernamentales.

\section{MATERIAL Y MÉTODO}

Se efectuó una búsqueda de la revisión sistémica de la literatura en las bases de datos Pubmed, Scielo y BVS (Biblioteca Virtual en Salud). Las palabras claves fueron Marcado, etiquetado, dentadura completa, prótesis dental total, prótesis dentaria, odontología forense, odontología legal. Los criterios de inclusión establecidos para el trabajo fueron los siguientes, artículos que describan las tres técnicas de marcado (Código QR, Tarjetas digitales e Incorporación de banda metálica), artículos de revisión de la literatura, artículos publicados entre los años 2012-2019, en idioma español e inglés. Los criterios de exclusión fueron artículos en los que se explicaba sobre otras técnicas de marcado y artículos anteriores al 2012. Se encontraron un total de 105 artículos, de los cuales fueron excluidos los que no tenían relación con nuestra investigación, quedando 16 artículos seleccionados. 


\section{REVISIÓN DE LA LITERATURA}

\section{Técnicas de marcaje de prótesis completa}

Existen diferentes tipos de marcado y etiquetado de prótesis dentales, según el proceso utilizado para su elaboración. Básicamente se distinguen dos clases: técnicas de marcado superficial y de marcado por inclusión. En la primera se realiza un grabado en la superficie externa de la prótesis, en el acrílico o en el metal, presenta la desventaja de favorecer la retención de residuos y no ser muy resistente a la abrasión o altas temperaturas. En la segunda se incorpora en la estructura de la prótesis una etiqueta o aditamento con la información del paciente (puede ser un código QR, una tarjeta de memoria, microchip o una banda metálica). Esta última técnica puede aplicarse durante la elaboración de la prótesis o cuando está terminada. Además, es más utilizada por los beneficios que brinda y ser más permanente. La localización idónea del marcado en el maxilar, es a nivel del paladar posterior y en la mandíbula en la pestaña lingual ${ }^{(2,11)}$.

Es recomendable que el marcado de la prótesis no debilite la estructura y sea estéticamente aceptable. Así mismo seria mejor si resistiera la acción del fuego o solventes.

\section{Marcado mediante un código QR}

Esta técnica consiste en la generación de un código 2D, el cual será impreso en tamaño de $10 \mathrm{x}$ $10 \mathrm{~mm}$ en papel fotográfico. La información a utilizar consistirá en el nombre completo, edad, sexo, ciudad, número de identificación del paciente. Luego de imprimir el código que tendrá forma cuadrada, este pasará por agua para evitar que la tinta se disperse, después el código será laminado con resina acrílica transparente. Mientras tanto se prepara las cavidades de las prótesis que tendrá $1 \mathrm{~mm}$ de profundidad. El código se localizará en la zona superior a nivel de la superficie palatina y en zona inferior a nivel de la pestaña distolingual. Para terminar, los códigos serán colocados en sus respectivos lugares donde se procederá a cubrirlos con resina acrílica transparentes para luego ser pulidas $^{(9,12)}$.

Rojas-Torres $\mathrm{J}$ et al, en su trabajo de investigación sobre actitudes, percepciones y preferencias de individuos de Temuco (Chile) sobre el marcado de prótesis dental, obtienen como resultado que el $90.56 \%$ de la población marcaria su prótesis con algún sistema, prefiriendo los códigos QR o tarjeta micro SIM ${ }^{(13)}$

Poovannan S, et al. (2016) valoró la capacidad de escaneo del código QR, en distintas profundidades de la lamina de acrílico. La sometió a ácido y a calor de hasta $370^{\circ} \mathrm{C}$. Ellos concluyeron que el código QR era un método de marcado de prótesis simple y eficaz ${ }^{(14)}$.

Pathak C et al. (2018) en la India, optaron por marcar las dentaduras empleando el código QR, las cuales fueron cargadas con información obtenida de la tarjeta Aadhaar (Número de Identificación) ${ }^{(16)}$. Seleccionaron un paciente al azar, cuando se terminó la elaboración de la prótesis, esta pasó por el proceso de marcaje, según el procedimiento explicado. Para poder acceder a la información almacenada en los códigos $\mathrm{QR}$, descargaron un programa de lectura de códigos en el celular, el cual al escanear el patrón de códigos permitía el acceso a la información de la persona ${ }^{(15)}$.

\section{Tarjetas digitales}

El sistema consiste en almacenar la información en una tarjeta SD a través de fotografías, textos, videos que podrán ser accedidos mediante un adaptador de memoria que podrá ser conectado a cualquier ordenador. Se prosigue con el tallado en la prótesis para lo cual se tomarán las medidas de la tarjeta. Estas serán colocadas en el modelo como guía. Cuando se termina de tallar, se coloca la tarjeta SD, la cual es forrada con cinta de embalaje que sirve como medio de protección. Se fabrica una lámina de cera que servirá como una tapa, la cual se coloca en la prótesis, para finalizar se procede con el sellado y pulido. Presenta ventajas: no interfieren con la masticación, la tarjeta SD es de bajo costo y no es necesario la capacitación para instalar el dispositivo ${ }^{(15)}$.

\section{Incorporación de banda metálica:}

Consiste en preparar una ranura de $1 \mathrm{~mm}$ de profundidad en la base de la prótesis. Este espacio será ocupado por una banda metálica que puede ser, lámina de titanio o níquel-cromo. Una vez colocada la banda, se cubre con acrílico transparente, luego se pule ${ }^{(16)}$. Se han realizado diversas pruebas para valorar su resistencia, una de ellas fue la prueba térmica, las bandas metálicas fueron expuestas a temperaturas altas de $800^{\circ} \mathrm{C}$, $1000^{\circ} \mathrm{C}$ y $1200^{\circ} \mathrm{C}$, el resultado obtenido fue el siguiente: el bloque acrílico fue destruido mientas que la banda metálica resistió ${ }^{(11)}$. Esta técnica presenta una gran resistencia al fuego, siendo esta su mayor ventaja. 
Tabla 1. Comparación de trabajos de investigación en los cuales utilizaron marcado de prótesis dental

\begin{tabular}{|c|c|c|c|c|}
\hline Articulo & Autor & Año & Objetivo & Conclusión \\
\hline $\begin{array}{l}\text { Sistema único de } \\
\text { identificación de } \\
\text { prótesis para todos } \\
\text { los ciudadanos } \\
\text { indios }\end{array}$ & $\begin{array}{l}\text { Pathak C, } \\
\text { Pawah S, Sikri } \\
\text { A, Rao }{ }^{(15)}\end{array}$ & 2018 & $\begin{array}{l}\text { Proponer una técnica de } \\
\text { marcado de prótesis dental } \\
\text { que proporcione una } \\
\text { identificación permanente, } \\
\text { única y global a todos los } \\
\text { ciudadanos indios que } \\
\text { usan Aadhaar, una } \\
\text { identidad universalmente } \\
\text { aceptada para ellos. }\end{array}$ & $\begin{array}{l}\text { Una tarjeta de Aadhaar es una } \\
\text { identificación oficial como } \\
\text { ciudadano indio. Las prótesis } \\
\text { dentales con códigos QR de } \\
\text { Aadhaar se pueden reconocer } \\
\text { fácilmente con la ayuda de una } \\
\text { cámara móvil. Todas las } \\
\text { autoridades encargadas de hacer } \\
\text { cumplir la ley deben enfatizar la } \\
\text { importancia del marcado de la } \\
\text { dentadura con el código / número } \\
\text { QR de la tarjeta Aadhaar, y debe } \\
\text { promoverse entre todos los } \\
\text { dentistas, para que sea un } \\
\text { procedimiento dental de rutina } \\
\text { obligatorio en todo el país. }\end{array}$ \\
\hline $\begin{array}{l}\text { Un caso de } \\
\text { identificación } \\
\text { forense y DPid: } \\
\text { ¿puede ser una } \\
\text { herramienta útil? }\end{array}$ & $\begin{array}{l}\text { Queiroz CL, } \\
\text { Bostock EM, } \\
\text { Santos CF, } \\
\text { Guimaraes MA, } \\
\text { Silva RH. } \\
\text {,citados por } \\
\text { Leão } 2017^{(17)}\end{array}$ & 2017 & $\begin{array}{l}\text { El objetivo de este estudio } \\
\text { fue mostrar el DPid como } \\
\text { una herramienta } \\
\text { importante de aplicación } \\
\text { potencial para resolver } \\
\text { casos con prótesis dental, } \\
\text { como el caso forense } \\
\text { reportado, en el que se } \\
\text { recibió un cráneo, prótesis } \\
\text { y registros dentales para } \\
\text { su análisis. }\end{array}$ & $\begin{array}{l}\text { Si la herramienta DPid estuvo } \\
\text { presente en este caso forense, } \\
\text { podría haberse resuelto sin } \\
\text { necesidad de un examen de ADN, } \\
\text { que confirmó la comparación } \\
\text { dental de los registros antemortem } \\
\text { y postmortem, y concluyó el caso } \\
\text { como una identificación positiva. }\end{array}$ \\
\hline $\begin{array}{lr}\text { Identificación } & \text { de } \\
\text { prótesis dentales } \\
\text { utilizando } & \text { el } \\
\text { número } & \text { de } \\
\text { identificación } & \\
\text { nacional individual } \\
\text { de Arabia Saudita: } \\
\text { Un r método } \\
\text { innovador r de } \\
\text { inclusión de } & \text { metal } \\
\text { fundido } & \end{array}$ & $\begin{array}{lr}\text { Bhardwaj } & \text { A, } \\
\text { Priya } & M S, \\
\text { Kannan } & S, \\
\text { Singh } & \text { S, } \\
\text { Ahmed } & \text { S, } \\
\text { Fadaleh } \mathrm{M}^{(16)}\end{array}$ & 2017 & $\begin{array}{l}\text { El objetivo fue encontrar } \\
\text { una técnica de } \\
\text { identificación de prótesis } \\
\text { fácil, menos costosa, } \\
\text { duradera y estandarizada }\end{array}$ & $\begin{array}{l}\text { La técnica propuesta es un } \\
\text { método de identificación fácil, } \\
\text { menos costoso, duradero, } \\
\text { radiográficamente visible y } \\
\text { estandarizado }\end{array}$ \\
\hline $\begin{array}{l}\text { Una evaluación in } \\
\text { vitro de la fiabilidad } \\
\text { de la técnica de } \\
\text { etiquetado de de } \\
\text { prótesis de código } \\
\text { QR }\end{array}$ & \begin{tabular}{l}
\multicolumn{2}{l}{ Poovannan S, } \\
Jain \\
Krishnan \\
Chandran V, \\
(14)
\end{tabular} & 2016 & 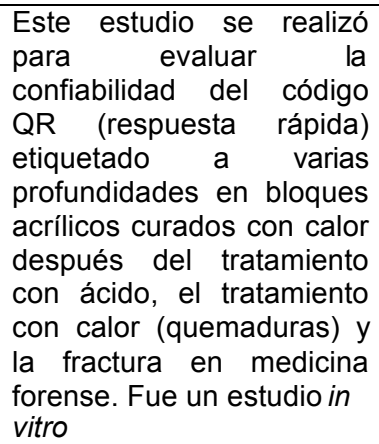 & $\begin{array}{l}\text { Dentro de las limitaciones del } \\
\text { estudio, se indicó que la técnica } \\
\text { del Código QR era confiable bajo } \\
\text { varias profundidades de lámina } \\
\text { acrílica, ácido y calor ( }\left(370^{\circ} \mathrm{C}\right) \text {. La } \\
\text { efectividad variaba en fracturas y } \\
\text { dependía del nivel de } \\
\text { distorsión. Este estudio sugiere } \\
\text { que el Código QR es un método } \\
\text { de etiquetado de dentadura eficaz } \\
\text { y más simple. }\end{array}$ \\
\hline $\begin{array}{l}\text { Marcado de } \\
\text { prótesis dentales } \\
\text { para identificación } \\
\text { forense con tarjeta } \\
\text { de memoria: una } \\
\text { técnica innovadora }\end{array}$ & $\begin{array}{l}\text { Lutha R, Arora } \\
\text { S, Sikri A, Rao } \\
\text { L. (18). }\end{array}$ & 2012 & $\begin{array}{l}\text { El propósito de este } \\
\text { estudio fue evaluar la } \\
\text { viabilidad del uso de una } \\
\text { tarjeta de memoria para la } \\
\text { identificación forense de } \\
\text { prótesis dentales. }\end{array}$ & $\begin{array}{l}\text { La incorporación de una tarjeta de } \\
\text { memoria en la base de la } \\
\text { dentadura artificial y su posterior } \\
\text { recuperación para la identificación } \\
\text { post mortem de víctimas de } \\
\text { asesinato, suicidio o en caso de } \\
\text { desastre como inundaciones, } \\
\text { accidentes de trenes, derrumbes } \\
\text { de edificios o terremotos, podría } \\
\text { convertirla en una herramienta } \\
\text { valiosa en las manos del dentista } \\
\text { para fines forenses. }\end{array}$ \\
\hline
\end{tabular}




\section{DISCUSIÓN}

El presente artículo de revisión de la literatura tuvo como finalidad demostrar que el marcado de la prótesis dental es importante para la identificación de las personas. Para lo cual se escogieron tres técnicas de marcado que pueden ser implementadas en el Perú. Poovannan S, et al. (2016) en su investigación indicaron que los códigos $Q R$, colocados a profundidades determinadas podían soportar condiciones ácidas y de calor $\left(370^{\circ} \mathrm{C}\right)^{(14)}$. Pathak C et al. (2018) utilizaron datos de la tarjeta Aadhaar y los almacenaron en los Códigos QR, para acceder a la información era necesario un aplicativo móvil ${ }^{(15))}$. Estos dos trabajos son importantes, ya que al finalizar demuestran la viabilidad de esta técnica. Lutha R, et al. (2012), en su trabajo indicaron que las tarjetas de memoria son llenadas con la información del paciente para luego ser colocadas en la prótesis dental. Asimismo, mencionaron que estos códigos pueden resistir temperatura de $400-600{ }^{0} \mathrm{C}$ por periodo de 30 minutos ${ }^{(18)}$. Atul Bhardwaj y et al (2017), utilizaron la técnica de banda metálica para el reconocimiento de personas, la cual presenta varias ventajas ${ }^{(16)}$. Las tres técnicas mencionadas con anterioridad se caracterizan por ser factibles de ser desarrolladas por los odontólogos en sus consultorios, presentan bajo costo y son estéticamente aceptables. Según los artículos encontrados, se evaluó la resistencia a altas temperaturas de calor, determinando que la técnica de incorporación de banda metálica, presenta mayor resistencia seguida de las tarjetas de memoria y códigos QR.

Las limitaciones que se presentaron en esta revisión, fueron que no se encontraron muchos trabajos relacionados al marcado de prótesis dental en América Latina, lo que hubiera favorecido a comparar las realidades entre países y como estas fueron aplicadas.

\section{Conclusión}

El marcado de prótesis dentales es importante para el reconocimiento de víctimas. Debería considerarse la aplicación de las técnicas de marcado prótesis, por las entidades gubernamentales, debido a su utilidad, bajo costo y accesibilidad.

Estas técnicas no solo pueden ser utilizadas en prótesis totales, sino también en prótesis parciales removibles, aumentado el rango de población que podría ser identificada por este medio.

Luego de realizar el marcado de las prótesis totales con Código QR y Micro SD, se asume que son técnicas sencillas de amplio almacenamiento, bajo costo y que pueden ser implementadas para beneficio de la población.

Considerando la importancia y viabilidad en la aplicación de estas técnicas se sugiere realizar investigaciones, con pruebas de resistencia y estudios en pacientes para valorar su funcionalidad, además, poder buscar nuevas formas de marcado. Se recomienda unificar protocolos de implementación de marcado de prótesis dental a fin incluir normativas que reglamenten su uso por las instituciones gubernamentales.

\section{DESCRIPCIÓN DE DOS TÉCNICAS}

De las tres técnicas mencionadas para marcar las prótesis totales, se eligieron dos de ellas para su confección, basándonos en su amplia capacidad de almacenamiento y bajo costo, lo cual haría mas factible su aplicación en nuestro país. Las técnicas elegidas fueron marcado de prótesis con microSD y con códigos QR.

Se valoró el tiempo desde el comienzo del tallado hasta el pulido final; la información para llenar las tarjetas de memoria y los códigos QR, se obtuvo del DNI (documento nacional de identidad), además se agregó el nombre y número telefónico de un familiar. Para la lectura de los códigos QR es necesario un aplicativo móvil y un lector de memoria para microSD. El costo del microSD en Perú bordea los S/. 24.00 un equivalente aproximado de $\$ 7.00$ y cada código QR impreso en papel fotográfico es de s/. 1.00 alrededor de \$0.3. La aplicación de esta técnica brinda resultados estéticamente aceptables y no interfiere con las funciones masticatorias. No es necesario una capacitación especial, pudiendo ser realizada por el odontólogo en su consultorio.

\section{Proceso de marcado de prótesis}

Se necesita un Código QR impreso, un microSD, Lector de memoria, cinta adhesiva transparente, resina transparente, acrílico de autocurado, fresas de carburo, piedras y pastas para pulir.

\section{Marcado mediante Código QR}

1. Se genera un código $Q R$, con datos adquiridos del DNI (Documento Nacional de Identidad), el cual presenta ocho dígitos que es diferente en cada ciudadano. La información brindada es Apellidos, Nombre, Fecha de nacimiento, dirección, estado civil. A esta información se agregaría número telefónico del paciente, además del nombre, apellido y número telefónico de un familiar cercano. 
2. El código $Q R$ es impreso en papel fotográfico, con las medidas de $10 \times 10$ milímetro. Luego es cubierto con cinta de embalaje para su protección. Figuras 1 y 2 .

3. El código $Q R$ será impreso en papel fotográfico, con las medidas de $10 \times 10$ milímetro. Ya impreso se procede a forrarlo con cinta de embalaje para su protección.
4. En la prótesis se talla una cavidad de $1 \times 1$ $\mathrm{x} 1 \mathrm{~cm}$., donde será colocada el código. Figura 3.

5. Se procede a colocar el código $Q R$ dentro de la cavidad, se cubre con resina trasparente de autopolimerización , para luego terminar con el pulido. Figuras 4 y 5 .

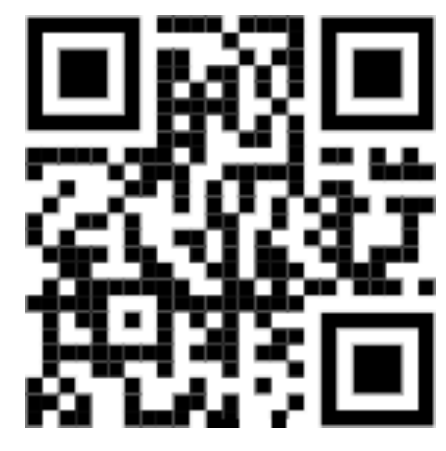

Figura 1. Código QR
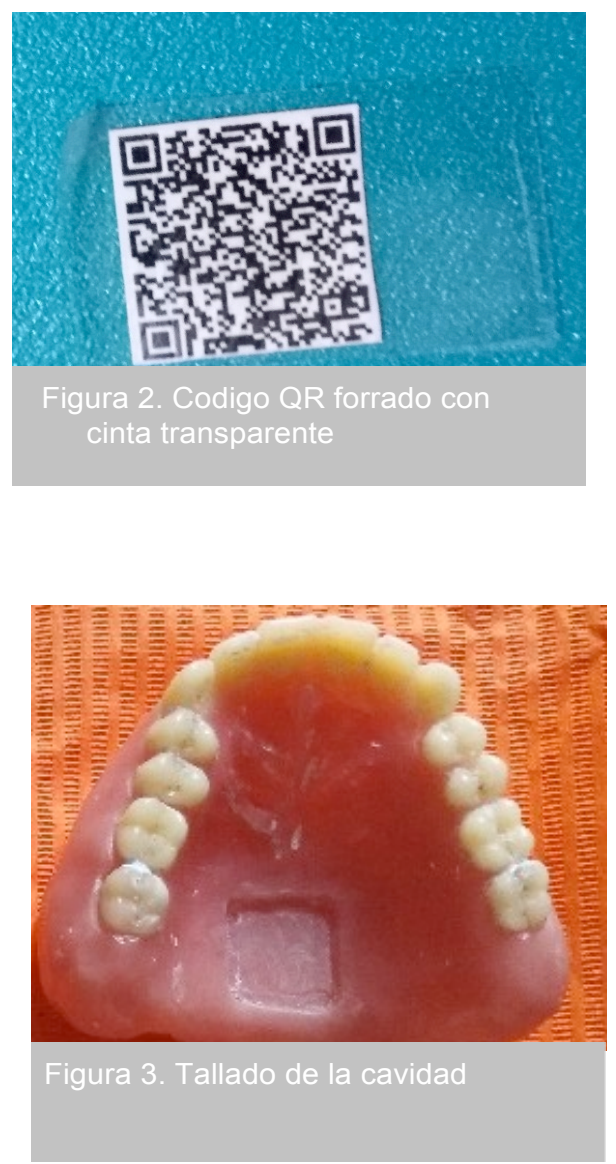

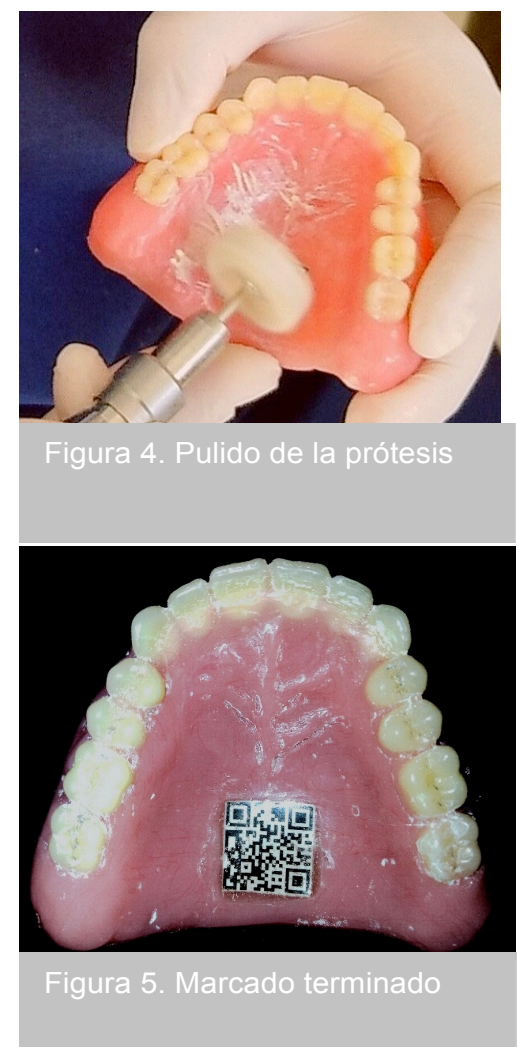




\section{Marcado mediante microSD}

En el mercado se puede encontrar diferentes marcas de microSD, con capacidad de almacenamiento diverso. Para esta demostración, se utilizó el de $4 \mathrm{MB}$, en el cual se guardó la información del paciente extraída del DNI, además se agregó el nombre y número telefónico de un familiar cercano. Figura 5. Para acceder a la información se empleará un lector de memoria, que podrá ser conectado a cualquier computador.

Al terminar la elaboración de la prótesis, se procede con el marcado para lo cual se toma
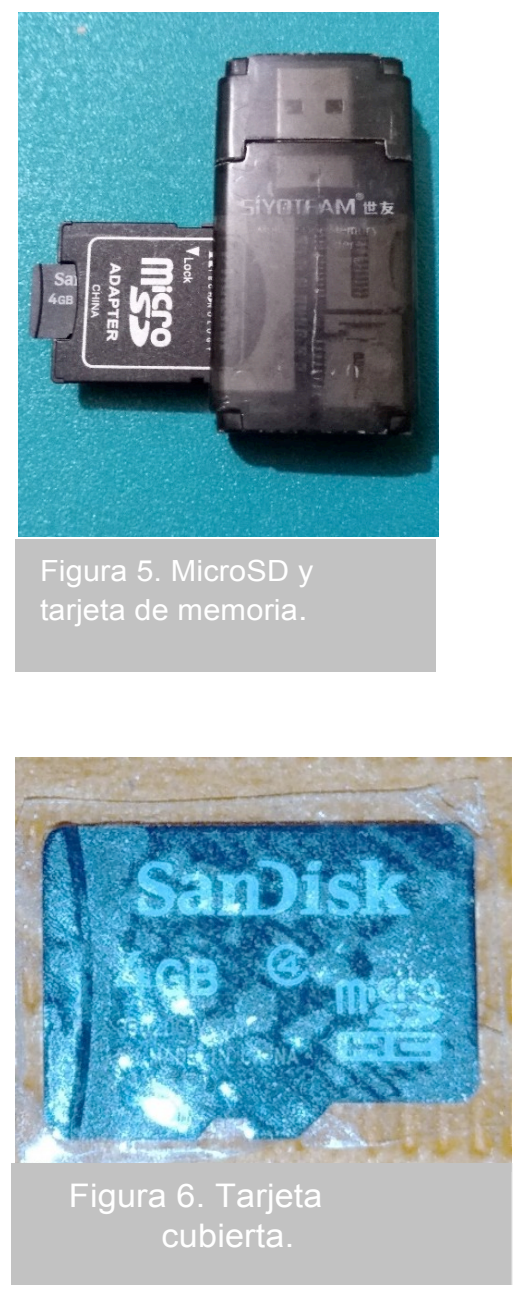

las medidas del microSD $15 \times 11 \times 1$ milímetros, a estas medidas se le aumenta $18 \times 14 \times$ 2 milimetros, sin debilitar la prótesis. Para lo cual se utilizará fresas de carburo.

Teniendo la información en el microSD, se procede a cubrirlo con cinta de embalaje, para protegerlo de polvos y líquidos. Figura 6.

Con el microSD cubierto, se procede a colocar en la cavidad realizada en la prótesis, luego se cubre con resina acrílica rosada de autopolimerización. Para terminar, se realiza el pulido. Figuras 7 y 8 .
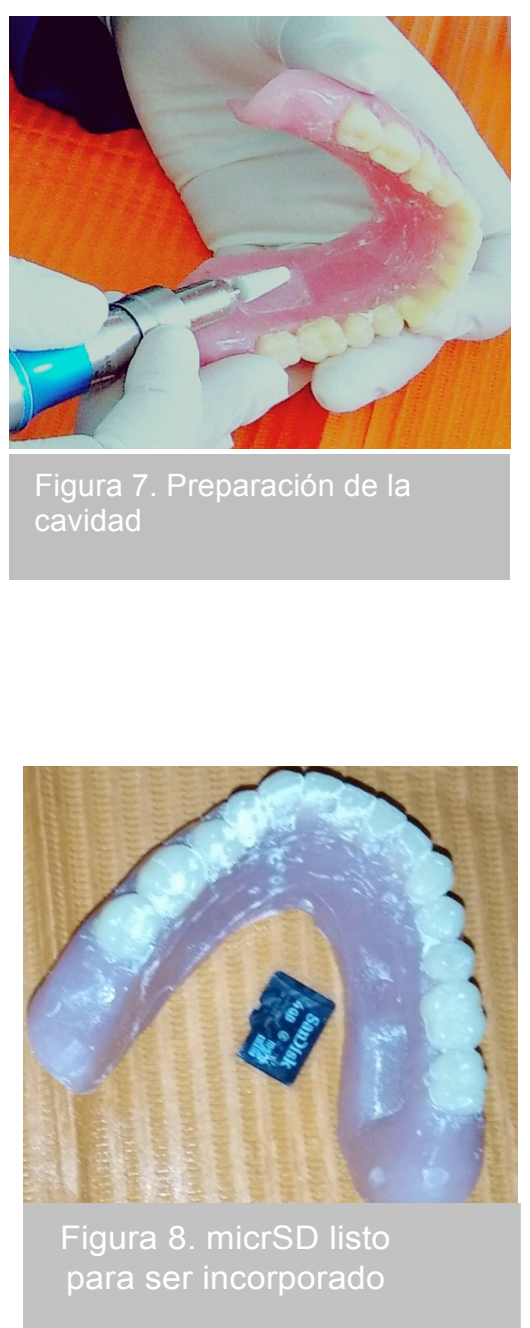
Contribuciones de autoría: GAS, JFG: Participaron en la revisión de la información, redacción y aprobación del artículo.

Fuente de financiamiento: Autofinanciado.

Conflictos de interés: No declararon conflictos de interés.

\section{REFERENCIAS BIBLIOGRÁFICAS}

1. Chugh A, Narwal A. Oral mark in the application of an individual identification: From ashes to truth.J Forensic Dent Sci.2017.May-Aug 9(2)5155.Disponible en :https://www.ncbi.nlm.nih.gov/pmc/articles/PMC5717 772/

2. Medina s, Vallejo D, Moreno F, Técnicas, Etiquetas y dispositivos de marcaje de prótesis dental empleados en odontología forense como medio de identificación.Univ Odontol.2014 (71): 19 - 28.

3. Para no olvidar: a 17 años del terrible incendio de Mesa Redonda. Perú 21.2018 Dic. Disponible en : https://peru21.pe/lima/olvidar-17-anos-terribleincendio-mesa-redonda-450070-noticia/

4. Mesa Redonda: Realizan vigilia a los 17 del trágico incendio. El comercio.2018 Dic. Disponible en : https://elcomercio.pe/lima/sucesos/mesa-redondarealizan-vigilia-17-anos-tragico-incendio-fotosnoticia-nndc-592392-noticia/

5. Baad R, Belgaumi U, Vibhute N, Kadashetti V, Chandrappa P, Gugwad S.Proposing national identification number on dental prostheses as universal personal identification code $-\mathrm{A}$ revolution in forensic odontology.J Forensic Dent Sci.2015 May- Ag; 7(2): 84-89.Disponible en : https://www.ncbi.nlm.nih.gov/pmc/articles/PMC4430 $580 /$

6. Rao Bathala L, Kumar Rachuri N, Rao Rayapati, Kondaka S.Prosthodonthics an "arsenal" in forensic dentristry.J Forensic Dent Sci.2016 (3): 173. Disponible https://www.ncbi.nlm.nih.gov/pmc/articles/PMC5210 107/

7. Phulari $R$, Rathore $R$, Jariwala $P$. Importance of prosthesis marking for human identification in forensic odontology.J Clin Diagn Res.2014 Nov;8 (11):1. Disponible en : https://www.ncbi.nlm.nih.gov/pmc/articles/PMC4290 266/

8. Sudheendra US, Sowmya K, Vidhi M, Shreenivas K, Prathamesh J. 2D barcodes: D novel and simple method for denture identification. J Forensic Sci. 2013; 58(1): 170-2.

9. Dineshshankar J, Venkateshwaran R, Vidhya J, Anuradha R, Pealin Mary G, Pradeep R,
Senthileappan.Denture Bar-coding: An innovative technique in forensic dentistry. J Pharm Biollied Sci.2015 Agt; 7(2): 350-353: Disponible en: https://www.ncbi.nlm.nih.gov/pmc/articles/PMC4606 618/

10. Kareker N, Aras M, Chitre V. A Review on Denture Marking Systems: A Mark in Forensic Dentistry.J Indian Prosthodont Soc.2014(1):4 - 13.Disponible en:

https://www.ncbi.nlm.nih.gov/pmc/articles/PMC4502 006/

11. Phulari R, Rathore R, Jariwala P, Kapuriya A, Shah A.Denture labels: Various types and their abilities to resist different assaults.J Forensic Dent Sci.2017 Sep- Dic; 9(3):175.Disponible en: https://www.ncbi.nlm.nih.gov/pmc/articles/PMC5887 643/

12. Basavanna J M, Jain A, Misra $S$ K. Denture barcoding in forensic dentistry: A future option.J Forensic Dent Sci. 2106 Jan-Apr; 8(1) 52-5. Disponible en https://www.ncbi.nlm.nih.gov/pmc/articles/PMC4799 $520 /$

13. Rojas-Torres J, Navarro-Cáceres P, Fonseca GM. Actitudes, percepciones y preferencias de individuos de Temuco (Chile)Sobre el marcado de la dentadura postiza. J Ciencia forense.2019 Jul;64(4):11871195.

14. Poovannan $S$, Jain $A$, Jalliah $C$, Krishnan $\mathrm{V}$,Chandran C.An In vitro evaluation of the reliability of QR code denture labeling technique: J Forensic Dent Sci.2106 Sep- Dic; 8(3):179.Disponible en: https://www.ncbi.nlm.nih.gov/pmc/articles/PMC5210 $117 /$

15. Pathak C, Pawah S, Sikri A, Rao L. Unique Denture Identification System for All India Nationals.Contemp Clin Dent.2018 Jun; 9(1)185 - 188.Disponible en: https://www.ncbi.nlm.nih.gov/pmc/articles/PMC6006 8721

16. Bhardwaj A, Priya MS, Kannan S, Singh S, Ahmed $S$, Fadaleh M.Denture identification using individual national identification number of Saudi Arabia: An innovative inclusion method of casted metal. J Forensic Dent Sci. 2017 Jan- Apr; 9():20-30. Disponible en: https://www.ncbi.nlm.nih.gov/pmc/articles/PMC5450 478/

17. Leão de Quiroz C, Bostock E, Ferreira A, Guimarães $M$, Alves da Silva R.A forensic identification case and DPid- can it be a useful tool?. J Appl Oral Sci.2017 May-Jun;25(3):347-353

18. Lutha R, Arora S, Meshram.Denture Marking for Forensic Identificacion Using Memory Card.J Indian Prosthodont Soc.2012 Dec;12(4):231-235. Disponible en: https://www.ncbi.nlm.nih.gov/pmc/articles/PMC3508 104/ 